\title{
XMS: Cross-Platform Normalization Method for Multimodal Mass Spectrometric Tissue Profiling
}

\author{
Ottmar Golf, ${ }^{1,3}$ Laura J. Muirhead, ${ }^{3}$ Abigail Speller, ${ }^{3}$ Júlia Balog, ${ }^{2,3}$ Nima Abbassi-Ghadi, ${ }^{3}$ \\ Sacheen Kumar, ${ }^{3}$ Anna Mróz, ${ }^{3}$ Kirill Veselkov, ${ }^{3}$ Zoltán Takáts ${ }^{1,3}$ \\ ${ }^{1}$ Institute for Inorganic and Analytical Chemistry, Justus Liebig University, Giessen, Germany \\ ${ }^{2}$ Waters Research Center, Budapest, Hungary \\ ${ }^{3}$ Department of Surgery and Cancer, Faculty of Medicine, Imperial College London, London, UK
}

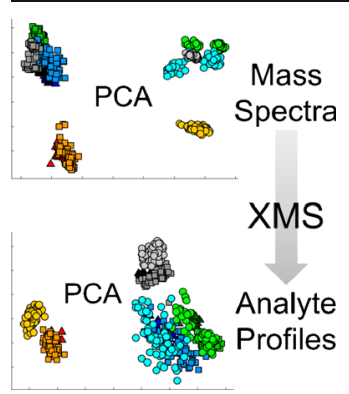

Abstract. Here we present a proof of concept cross-platform normalization approach to convert raw mass spectra acquired by distinct desorption ionization methods and/ or instrumental setups to cross-platform normalized analyte profiles. The initial step of the workflow is database driven peak annotation followed by summarization of peak intensities of different ions from the same molecule. The resulting compoundintensity spectra are adjusted to a method-independent intensity scale by using predetermined, compound-specific normalization factors. The method is based on the assumption that distinct MS-based platforms capture a similar set of chemical species in a biological sample, though these species may exhibit platform-specific molecular ion intensity distribution patterns. The method was validated on two sample sets of (1) porcine tissue analyzed by laser desorption ionization (LDI), desorption electrospray ionization (DESI), and rapid evaporative ionization mass spectrometric (REIMS) in combination with Fourier transformationbased mass spectrometry; and (2) healthy/cancerous colorectal tissue analyzed by DESI and REIMS with the latter being combined with time-of-flight mass spectrometry. We demonstrate the capacity of our method to reduce MS-platform specific variation resulting in (1) high inter-platform concordance coefficients of analyte intensities; (2) clear principal component based clustering of analyte profiles according to histological tissue types, irrespective of the used desorption ionization technique or mass spectrometer; and (3) accurate "blind" classification of histologic tissue types using cross-platform normalized analyte profiles.

Key words: Mass spectrometry, Cross-platform normalization, Algorithm, Correlation, Desorption ionization mass spectrometry, Lipids, Desorption electrospray ionization, Rapid evaporative ionization mass spectrometry, Laser desorption ionization, Principal component analysis, k-Nearest neighbor, Peak annotation

Received: 19 May 2014/Revised: 1 September 2014/Accepted: 2 September 2014/Published online: 8 November 2014

\section{Introduction}

$\mathrm{T}$ he development of mass spectrometric tissue profiling techniques has gained significant momentum within the last two decades [1]. Mass spectrometry imaging (MSI) is rapidly advancing within this field, enabling simultaneous determination of hundreds to thousands of chemical species within a single tissue sample in a spatially resolved manner. Well established techniques for MSI of biological tissue in-

Electronic supplementary material The online version of this article (doi:10.1007/s13361-014-0997-6) contains supplementary material, which is available to authorized users.

Correspondence to: Kirill Veselkov; e-mail: kirill.veselkov04@imperial.ac.uk, Zoltán Takáts; e-mail: z.takats@imperial.ac.uk clude SIMS [2], desorption electrospray ionization (DESI) [3], matrix assisted laser desorption/ionization (MALDI) [4, 5], and laser desorption/ionization (LDI) among others. These techniques capture lipid species in the low molecular weight range, which have been shown to be closely associated with histologic or histopathologic tissue types [6-10]. Thus, MSI has the potential to provide fully automated, MSbased tissue identification systems for next generation chemical histology. Rapid evaporative ionization mass spectrometry (REIMS) technology has recently been developed primarily for intra-operative mass spectrometric characterization of tissues. The technique primarily relies on the detection of high abundant ionized lipid species produced by electrosurgical tissue dissection [11]. The associated tissue identification algorithm uses large-scale histologically 
assigned spectral libraries, built by REIMS analysis of ex-vivo tissue samples, which are utilized for machine learning-based identification of unknown tissue features. The REIMS approach suffers from poor spatial resolution and inefficient utilization of precious clinical samples; furthermore, the essential histologic validation may be hindered by the destructive nature of the REIMS technique. In contrast, MSI methods provide reliable spatial localization of these chemical species at a resolution on tissue of $7 \mu \mathrm{m}$ for MALDI [12] and $35 \mu \mathrm{m}$ for DESI [13], while being largely nondestructive, leaving intact tissue morphology behind. DESI MSI detects similar chemical species to REIMS; however, due to the different ionization mechanisms, mass spectral profiles are markedly different. Theoretically, DESI datasets acquired at histologically acceptable spatial resolution could be utilized to populate the large scale spectral databases required for in-vivo REIMS based tissue identification.

The major challenge lies in the mechanistic differences between desorption ionization methods and instrumental platforms, which introduce platform-specific variation to the datasets. These factors sufficiently hinder the construction of a REIMS tissue identification system using data collected by MSI approach.

To date, no cross-platform normalization algorithm has been developed for the reduction of platform-specific variation for combining mass spectral data obtained by different ionization methods and mass spectrometers. In turn, a large scale MS driven tissue identification system requires a histologically assigned database using fused, platform-independent spectral data. Several cross-platform normalization approaches have recently been developed in the field of transcriptomics [1421] with the aim of combining multiple datasets to increase sample size for improved statistical analysis. These approaches utilize a single expression per gene to derive cross-platform normalization gene- or gene cluster-specific factors to bring intensities from one platform in line with the other. These methods are not directly applicable to mass spectrometric data, since molecular ion patterns are specific to the ionization method and one species can often be associated with multiple ion signals. Hence, the cross-platform normalization methods for mass spectrometry require data preprocessing in order to obtain a cumulative intensity value for each analyte species, followed by application of cross-platform normalization procedures. To date, approaches using analyte-dependent normalization factors have been reported for LC-experiments [22] and MALDI imaging data (Tissue Extinction Coefficient, TEC) [23] processing. Also, independent MS platform integration for de novo protein sequencing has been reported by fusing top-down and bottom-up mass spectra to a common spectral vector [24].

In the present study, we propose a cross-platform normalization method (XMS) for MSI (DESI, LDI) and in-vivo MS (REIMS and related techniques) data. The XMS method comprises database driven analyte peak annotation and summation of all signals related to a single analyte to obtain a single intensity measure for each analyte species. This is followed by derivation of cross-platform normalization factors to bring analyte intensities from one platform to a common scale with the other. The method assumes (1) that there is a significant overlap between analyte sets detected by different MS platforms across tissue types, and (2) that different spectral peaks corresponding to the same molecule exhibit similar MS behavior. We demonstrate the capacity of our method to reduce MSplatform (e.g., REIMS, LDI, DESI with different kinds of mass analyzers) specific variation therefore allowing clear clustering of analyte profiles according to histological tissue types, irrespective of the used desorption ionization technique or mass spectrometer and accurate "blind" classification of histologic tissue types using cross-platform normalized analyte profiles.

\section{Materials and Methods}

\section{Chemicals and Sample Treatment}

HPLC grade water, hexane, isopropanol, chloroform, and methanol were purchased from Sigma-Aldrich (Steinheim, Germany), ammonium hydroxide was purchased from Merck KGaA (Darmstadt, Germany).

For the analysis of human samples, ethical approval was obtained from the South East London National Research Ethics Committee (Study ID 11/LO/1686).

Porcine sample collection was performed in two batches. Nine porcine organs consisting of three kidneys, brains, and livers (group 1) were obtained at the local slaughterhouse $2-3 \mathrm{~h}$ after animals' extermination. Two months later, another nine organs were obtained using similar protocol (group 2). The analyzed tissue types were kidney cortex, kidney medulla, brain grey matter, and liver. LDI and REIMS did not produce acceptable signal stability for brain white matter; therefore, this tissue types was omitted from the current study. For DESI analysis, 29 colorectal samples from 21 patients, and for REIMS analysis, another 15 samples from 12 patients were collected from surgical resection specimens and stored at $80^{\circ} \mathrm{C}$ before processing. Analyzed tissue types were healthy mucosa and cancerous mucosa.

For DESI analysis of porcine samples, tissues were cryosectioned using a cryotome (HM 525 cryostat, Thermo Scientific, Dreieich, Germany), and the $20 \mu \mathrm{m}$ thick sections were thaw-mounted onto glass slides. The samples were stored at $-80^{\circ} \mathrm{C}$. For LDI and REIMS analysis, bulk tissue samples were stored at $-20^{\circ} \mathrm{C}$. Samples were thawed at room temperature prior to mass spectrometric analysis. For DESI analysis of colorectal tissue, samples were sectioned to $10 \mu \mathrm{m}$ thickness.

In case of nanospray analysis, three tissue extracts were prepared in three parallels by homogenization of tissue specimens in a mixture of hexane:isopropanol 3:2. The suspension was filtered by a $5 \mu \mathrm{m}$ pore diameter PTFE filter, diluted with methanol, and then analyzed with nano-ESI-MS/MS. The software LipidXplorer [25] was used for lipid identification.

For sensitivity and specificity determination of peak annotation, L- $\alpha$-phosphatidylethanolamine from egg yolk (SigmaAldrich, Gillingham, UK) was solved in methanol:chloroform 
2:1 and then evenly applied on a glass slide. DESI analysis was carried out after solvent evaporation.

\section{Instrumentation}

DESI analysis of porcine samples was carried out using an inhouse built ion source. The incident angle of the solvent spray (methanol:water $1: 1$ containing $1 \% \mathrm{NH}_{3}$ ) was set to $80^{\circ}$. The distance from sprayer tip to sample and the distance between sprayer tip and MS inlet capillary were set to 3 and $8 \mathrm{~mm}$, respectively. Electrospray voltage was set to $4.5 \mathrm{kV}$. Samples were moved at a velocity of $100 \mu \mathrm{m} / \mathrm{s}$ with a solvent flow rate of $2 \mu \mathrm{L} / \mathrm{min}$. Colorectal sections were imaged at $75 \mu \mathrm{m}$ pixel size. Methanol:water $(95: 5 \mathrm{vol} / \mathrm{vol})$ was used as electrospray solvent with a flow rate of $1.5 \mu \mathrm{L} / \mathrm{min}$ at a nitrogen gas pressure of 4 bar. Following DESI analysis, sections were stained with H\&E for comparison of tissue architecture between the stained- and MSimage.

REIMS analysis of porcine samples was performed by using a RadioSurg 2200 (Meyer-Haake, Wehrheim, Germany) electrosurgical unit. Power in monopolar cutting mode was set between 40 and $60 \mathrm{~W}$. The aerosol formed by electrosurgical dissection was transferred to the mass spectrometer using a $2 \mathrm{~m}$ long PTFE tubing (Fluidflon PTFE tubing; LIQUID-scan GmbH Co. KG, Überlingen, Germany) with $1 / 8^{\prime \prime}$ outer diameter and 1/16" inner diameter. A Venturi pump (VAC 100; Parker Hannifin, Veriflo Division, Warwick, UK) was operated at a nitrogen inlet pressure of $5 \mathrm{bar}$ in order to establish sufficient suction force for aerosol transport. Exhaust of the Venturi pump was directed orthogonally onto the inlet of the mass spectrometer. A similar REIMS setup is described in detail elsewhere [26]. REIMS analysis of colorectal samples was performed at 30 $\mathrm{W}$ cutting mode and 2 bar gas pressure.

LDI analysis was performed using a frequency-tripled Nd:YAG laser (Brilliant; LOT-Oriel, Darmstadt, Germany, $\lambda=$ $355 \mathrm{~nm}$ ) with $10 \mathrm{~mJ}$ pulse energy, $4 \mathrm{~ns}$ pulse width, $20 \mathrm{~Hz}$ repetition rate, and a laser fluence of $2.5 \mathrm{~J} / \mathrm{cm}^{2}$. The desorbed aerosol was transferred to the mass spectrometer using a $40 \mathrm{~cm}$ long PTFE tubing (Fluidflon PTFE tubing; LIQUID-scan GmbH Co. KG, Überlingen, Germany) with $1 / 8^{\prime \prime}$ outer diameter and 1/16" inner diameter. The Venturi pump (VAC 100; Parker Hannifin, Veriflo Division, Warwick, UK) was operated at a nitrogen inlet pressure of 2.5 bar and the exhaust of the Venturi pump was directed orthogonally onto MS inlet. Since the laser falls into the class IV safety category, it has to be handled with extremely high caution, and appropriate safety goggles (LASERVISION GmbH, Fürth, Germany, matching for laser wavelength and energy) have to be worn as long as the laser is under power. Our laboratory followed the German Laser Safety Regulations (GUV-I 832).

Nano-ESI experiments were carried out using the standard NSI source of the mass spectrometer at $1.2 \mathrm{kV}$ electrospray voltage.

For DESI, LDI, REIMS, and nano-ESI experiments of porcine samples, high-resolution mass spectrometry was performed on a Thermo LTQ Orbitrap Discovery (Thermo Fisher Scientific Inc., Bremen, Germany). DESI analysis of colorectal samples was performed on a Thermo Exactive instrument (Thermo Fisher Scientific Inc., Bremen, Germany). REIMS analysis of colorectal samples was performed on a Waters Xevo G2-S Q-TOF instrument (Waters Micromass, Manchester, UK). Nano-ESI MS/MS experiments were carried out using high energy collision dissociation (HCD) with an isolation width of $2 \mathrm{~m} / \mathrm{z}$ units for the precursor ion. Additional DESI experiments from porcine group 2 tissues were carried out on a Thermo LTQ FT Ultra (Thermo Fisher Scientific Inc., Bremen, Germany). Experiments were performed in negative ion mode in the mass range of $600-1000 \mathrm{~m} / z$.

\section{Data Processing}

Cross-Platform Normalization Method The proposed crossplatform normalization workflow is summarized in Figure 1. It was developed based on the porcine data. XMS is logically divided into training and application phases. The training phase requires the acquisition of mass spectra of the same biological sample set on two or more distinct analytical platforms (referred to as a "reference" platform and a "test" platform(s) later in the text). The outcome of the training phase is a vector of analyte-specific normalization factors that account for intensity biases between analytical platforms. This cross-platform normalization vector is subsequently used in the application phase to transform analyte intensities from the test platform to a common scale of the reference platform. For the application of XMS to more than two platforms, one platform has to be defined as the reference platform and all other platforms have to be individually normalized to the reference.

Training and application phases share the following initial steps of data processing: (1) raw analytical signal processing, (2) database driven analyte peak annotation, and (3) analyte peak summation.

In the first step, real, ion-associated peaks are identified in raw (continuum) mass spectra. The mass spectra were initially interpolated with a resolution based on the minimum $\mathrm{m} / \mathrm{z}$ difference between two digitally recorded signals (e.g., $0.0056 \mathrm{Da}$ in the case of LTQ Orbitrap Discovery) to preserve high mass accuracy, and five representative spectra of the same tissue specimen were averaged. The representative $\mathrm{m} / \mathrm{z}$ ratio for a given peak was determined by the location of the peak's maximum intensity. The peak integrals were calculated using the trapezoidal rule [27]. Mass drift was corrected by linear internal calibration with respect to a reference library of molecular ion species with known $m / z$ values. Preprocessing of REIMS colorectal samples included additional steps being denoising by excluding all peaks smaller than the median plus four times the intensity standard deviation of the mass spectrum and correction of peak shapes by Savitzky-Golay smoothing.

In the second step, the automated annotation of mass spectral signals to specific molecular ion species was carried out using a home-built, high mass accuracy analyte database. It was populated with ions identified from tissue extracts by nano-ESI tandem mass spectrometry. In total, the database 


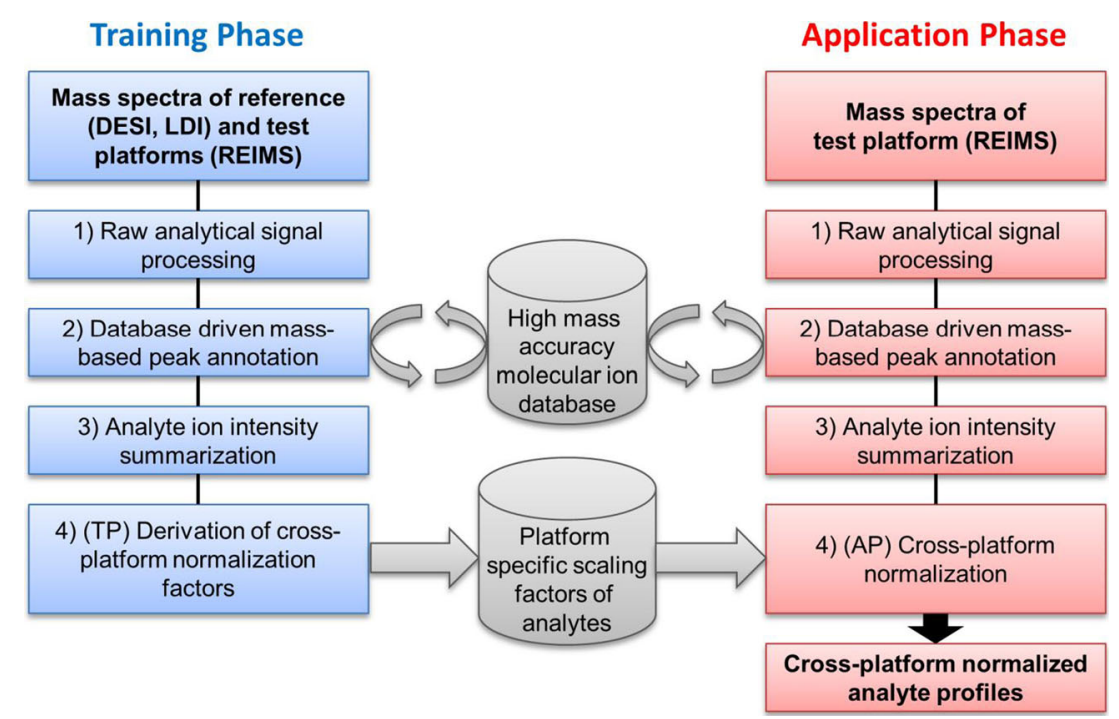

Figure 1. Cross-platform normalization method workflow for obtaining cross-platform normalized analyte profiles. Analyte specific normalisation factors obtained in the training phase are utilized to convert analyte intensities between platforms in the application phase

consists of 706 ion species from 537 analytes, including adducts and thermal decomposition ions such as $[\mathrm{M}-\mathrm{H}]^{-}$, $[\mathrm{M}+\mathrm{Cl}]^{-},\left[\mathrm{M}-\mathrm{NH}_{4}\right]^{-},[\mathrm{M}+\mathrm{Na}-2 \mathrm{H}]^{-},[\mathrm{M}+\mathrm{K}-2 \mathrm{H}]^{-}$and $[\mathrm{M}-$ $\left.\mathrm{H}_{2} \mathrm{O}-\mathrm{H}\right]^{-}$. The mass spectral peaks were matched to database molecular ion species when the $\mathrm{m} / \mathrm{z}$ difference between database and spectral peaks was less than a predetermined threshold value of $8 \mathrm{ppm}$ ( $30 \mathrm{ppm}$ for Xevo data). If several database molecular ion species were matched with a given peak, it was assigned to the candidate molecular ion with the smallest mass deviation.

In the third step, all identified intensities associated with a given molecular species were added up to obtain its cumulative intensity.

In the training phase, the cross-platform normalization factor for a given analyte is derived by dividing its mean level across all profiles of the test platform to that of the reference platform.

In the application phase, the derived vector of analytespecific normalization factors is utilized to convert analyte intensities of the test platform to a common scale of the reference platform.

\section{Cross-Platform Normalization Method Diagnostics and Validation}

Mean-Mean Plots and Inter-Platform Concordance Meanmean analyte scatter-plots were generated by plotting the mean levels of a common set of analytes for a given tissue type between a pair of analytical platforms. The positive correlation of tissue-specific analyte measurements between two analytical platforms will result in a near diagonal scatter along the ideal concordance line of pair-wise analytes intensity values (i.e., a tissue type-associated increase in the cumulative intensity of a given analyte on one platform positively correlates with its increase on the other platform). The quantitative measure of the inter-platform concordance of analytes levels was defined as

$$
\rho_{c}=\frac{2 \rho \sigma_{t e s t} \sigma_{r e f}}{\sigma_{\text {test }}^{2}+\sigma_{r e f}^{2}+\left(\mu_{\text {test }}-\mu_{r e f}\right)^{2}}
$$

where $\rho_{c}$ is the concordance correlation coefficient [28] and $\rho$ is Pearson's correlation coefficient, which is calculated from the mean intensity values $\mu_{\text {test }}$ ref of each tissue type and platform for test or reference platform. $\sigma_{\text {test } / r e f}$ is the standard deviation of the mean intensity values of $\mu_{\text {test/ref }}$. Concordance correlation coefficient measures the variation from the concordance line through the origin and explains the degree of agreement between the mean intensity values across two platforms. A high concordance correlation coefficient close to the value 1 indicates high agreement of compared spectral profiles, while a low coefficient close to the value 0 resembles low agreement.

Unsupervised Clustering via Principal Component Analysis (PCA) PCA was used to map multidimensional MS data into an uncorrelated set of components capturing the majority of variation in the data set. Graphical representations of the first few components were used to explore the overall similarities/ differences in analyte profiles between tissue specimens and analytical platforms. To ensure that the derived principal components explained systematic variation is not attributable to noise, $(10 \times 10)$ bi-cross validation was performed [29]. For bi-cross validation plot, see Supplemental Information 
Figure S-9. The variance captured in bi-cross validation was used as an indicator of model robustness $\left(\mathrm{R}_{\mathrm{cv}}\right)$. The successful cross-platform normalization method is expected to result in clear clustering of analyte profiles on the PC map according to sample types (e.g., tissue types), irrespective of the used desorption ionization technique or mass spectrometer.

Distance-Based k-Nearest Neighbor (nn) Classification The 3-nn classification algorithm was used to classify porcine spectral data acquired on one platform into tissue types based on the training spectral data acquired on the other platform. The Euclidean distance was employed to calculate overall spectral similarities or differences. Confusion matrices were used to show the accuracy of a classification result. Following the successful cross-platform normalization, the analyte profiles are expected to be classified into the correct sample type, irrespective of the used desorption ionization technique or mass spectrometer.

Support Vector Machine Classification Colorectal samples were classified using support vector machines (SVM, LIBSVM library [30]) with radial kernel type and leave-onepatient-out cross validation.

\section{Dataset Description}

The porcine dataset used for the purposes of this study is explained in Table 1. ProteoWizard's MSConvert (ver. 3.0.4468 64-bit) was used to transform Thermo Xcalibur and Waters .raw files/folders of porcine and REIMS colorectal samples to mzXML format using 64-bit precision for $\mathrm{m} / \mathrm{z}$ and intensity dimension [31]. Colorectal DESI images were converted to imzML file format and co-registered with $\mathrm{H} \& \mathrm{E}$ stained sections for detection of mucosa tissue type by a histologist [10]. Mass spectra from the selected regions of interest (healthy and cancerous mucosa) were subjected to XMS. The mzXML and imzML files were imported into MATLAB (R2013a V 8.1.0.604) for subsequent analysis and processing.

For the porcine dataset, the reference platform group consists of DESI, LDI, and REIMS Orbitrap spectral data, whilst the test platform utilizes REIMS Orbitrap mass spectra. The training phase was carried out twice: (1) with REIMS and DESI Orbitrap reference set data, and (2) with REIMS and LDI Orbitrap reference set data. During the application phase, derived cross-platform normalization factors were applied to the REIMS Orbitrap test dataset to adjust for analyte specific intensity biases between the DESI/LDI and REIMS platforms.

DESI FT-ICR sample size was chosen to be smaller compared to the other sample sets to show the ability of XMS to normalize unbalanced class sizes.

\section{Results and Discussion}

\section{Cross-Platform Normalization Method}

Although mass spectrometric tissue profiling techniques produce different raw mass spectral patterns as demonstrated by Figure 2, there is substantial overlap regarding the chemical species detected by the different techniques, for example in the case of REIMS and DESI (Table 2). In this case, the observed differences are mostly associated with the different mechanisms of ion formation. The DESI ionization mechanism comprises analyte extraction from the tissue surface followed by multiply charged droplets undergoing an electrospray-like ionization scenario $[32,33]$. In contrast, in the case of REIMS the tissue itself is converted into an aerosol by Joule-heating [34]. The major differences in the spectra are attributable to the intrinsic thermal degradation occurring during REIMS process, as it is clearly demonstrated by the phosphatidylethanolamine species giving $[\mathrm{M}-\mathrm{H}]^{-}$type ions in case of DESI and [M$\left.\mathrm{NH}_{4}\right]^{-}$type ions in case of REIMS (see Supplemental Information FigureS-1).

Significant overlap within the chemical species detected by each ionization technique is the basis for successful crossplatform normalization. Table 2 demonstrates that the overlap in chemical species detected by REIMS and DESI exceeds $60 \%$. The differences are primarily associated with the sensitivity of each individual method. However, due to molecular ion pattern differences between these platforms the spectra initially need to be preprocessed to obtain chemical profiles (See steps 1-3 in the method description). All parameters within the workflow have been optimized to achieve successful cross-platform normalization. However, it is important to note that changes in sampling parameters (i.e., spray voltage, laser fluence, solvent composition) might have an impact on ionization efficiency. Hence, similar sampling conditions need to be used for obtaining reproducible mass spectral profiles. Several mass spectra from the same tissue sample were averaged in order to increase signal to noise ratio. This led to improvement

Table 1. Number of Mass Spectra Used in This Work. Four Porcine Tissue Types are Considered for Each Desorption Method: Kidney Cortex, Kidney Medulla, Liver, and Brain Grey Matter

\begin{tabular}{llllll}
\hline Desorption method & Mass analyzer & Mass spectra per tissue type & Total number of mass spectra & Data group & Sample collection group \\
\hline DESI & Orbitrap & 300 & 1200 & Reference set & Group 2 \\
DESI & FT-ICR & 100 & 400 & Test set & Group 2 \\
LDI & Orbitrap & 300 & 1200 & Reference and test set & Group 2 \\
REIMS & Orbitrap & 300 & 1200 & Reference set & Group 2 \\
REIMS & Orbitrap & 300 & 1200 & Test set & Group 1 \\
\hline
\end{tabular}



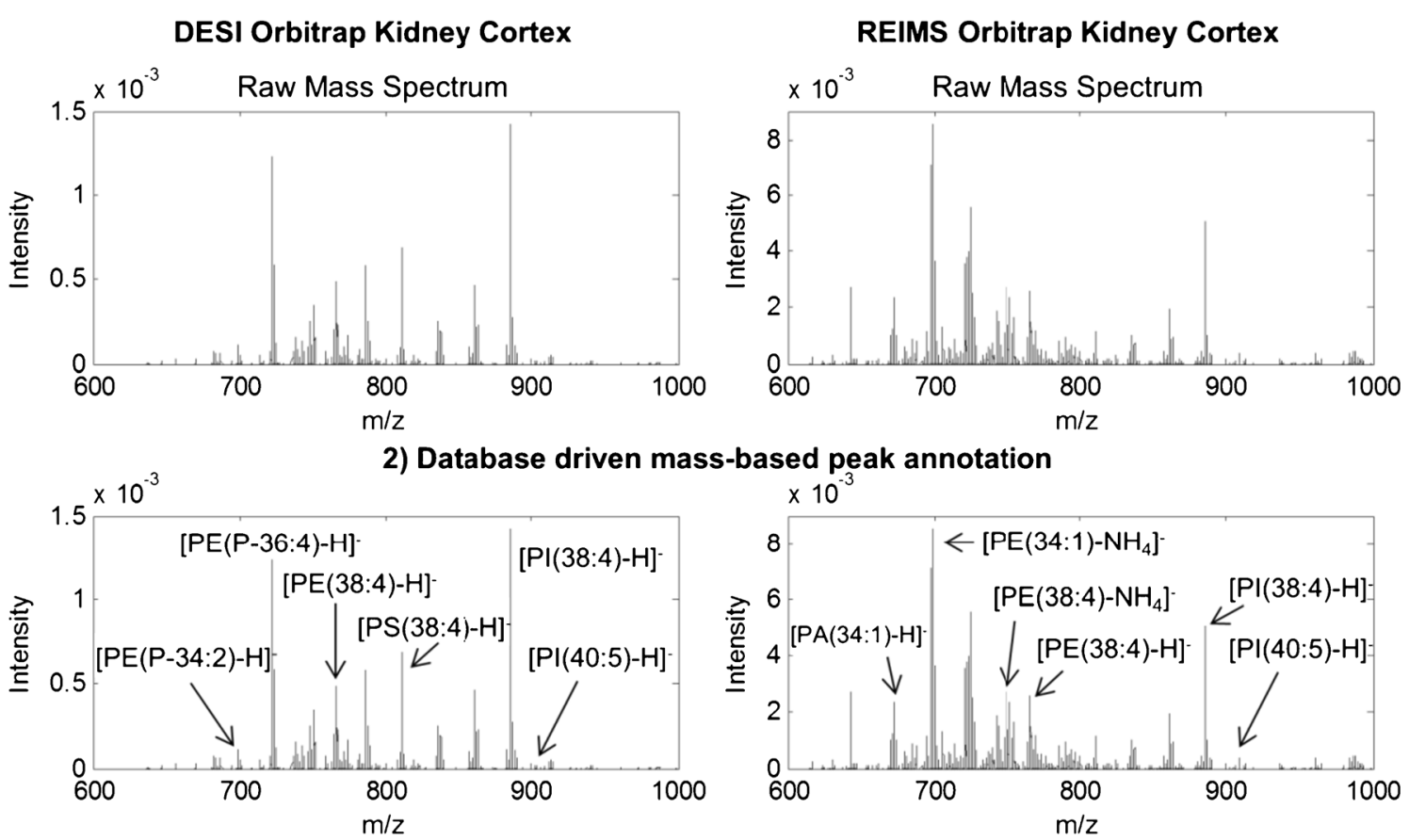

3) Analyte ion intensity summarization
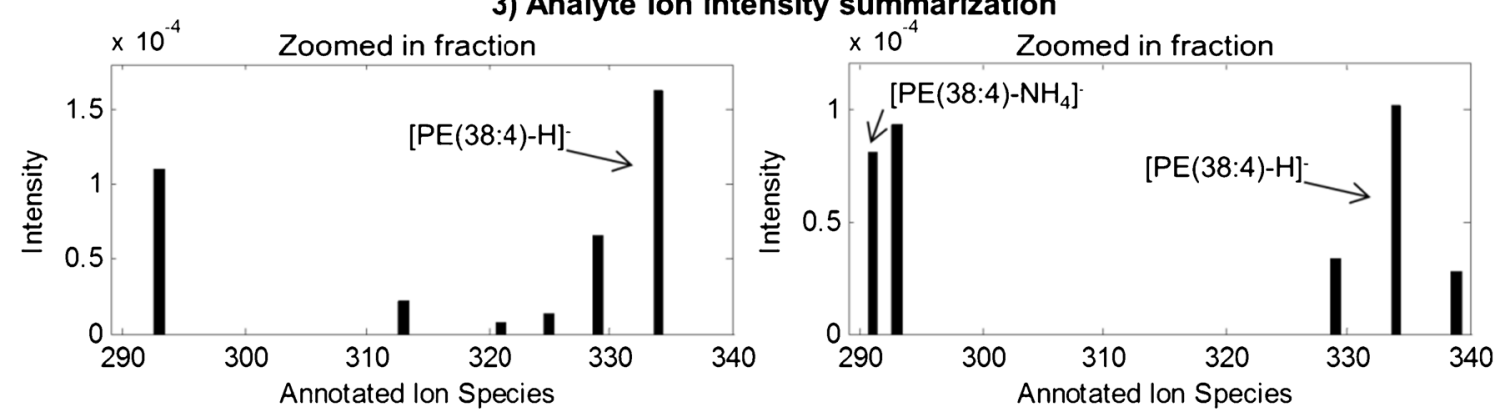

4.2) Cross-platform normalization
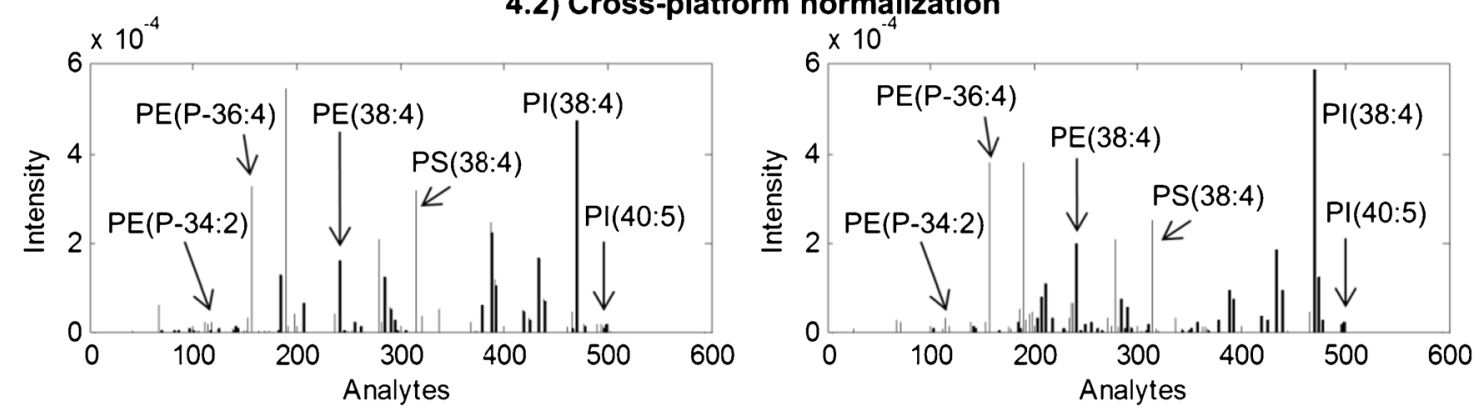

Figure 2. Porcine analyte profiles at different stages of the XMS processing workflow. $\mathrm{PE}=$ phosphatidyl-ethanolamine, $\mathrm{PA}=$ phosphatidic acid, $\mathrm{PS}=$ phosphatidyl-serine, $\mathrm{P}=$ Plasmalogen. For more details, see text

Table 2. Percentage of a Common Set of Analytes Present in both DESI/LDI and REIMS Porcine Spectral Data. Only Analytes Present in at Least $10 \%$ of the Mass Spectra of Each Tissue Type Were Included

\begin{tabular}{lll}
\hline & \multicolumn{2}{l}{ Overlaping analytes $/ \%$} \\
\cline { 2 - 3 } \cline { 2 - 3 } DESI - REIMS & LDI - REIMS \\
\cline { 2 - 3 } Kidney cortex & 64 & 39 \\
Brain grey matter & 65 & 38 \\
Liver & 60 & 51 \\
Kidney medulla & 62 & 44 \\
\hline
\end{tabular}

of data quality, where certain ionization methods (LDI) or tissue types (kidney medullary tissue) produced mass spectra with relatively low total ion counts (Supplemental Information Table S-1). Averaging five spectra from a single tissue sample was found to be optimal in this case, as was determined by evaluating the unknown REIMS spectra classification performance for different numbers of spectra averaged (Supplemental Information Figure S-10). DESI analysis of phosphatidyl-ethanolamine standard was used to determine the sensitivity and specificity of peak annotation. The best compromise between high specificity and good sensitivity 
(for values, see Supplemental Information Figure S-5) for matching analyte with the corresponding peak in the database was achieved with a mass deviation parameter of $8 \mathrm{ppm}$. The chosen mass range of $m / z=600$ to 1000 was found to be sufficient for accurate tissue identification using REIMS and DESI $[35,36]$. The different ionization techniques produce complex mass spectra from biological tissue samples, especially in the lower mass range where solvent and other peaks of numerous different molecule classes are present. Therefore, the number of possible ions/ion-fragments per peak would introduce a significant number of false positive peak annotations $[37,38]$. Also, the built-up of a high mass accuracy compound database for that mass range would be difficult in the first place. Still, database-driven peak annotation has the advantage that only peaks of analytes present in the database get annotated, while noise or unwanted fragment ions do not contribute to the classification. However, more sophisticated metabolite peak annotation strategies such as isotopic pattern interpretation may, in future, be incorporated into this pipeline to reduce the frequency of false annotations.

Owing to the fact that an ionization method can produce several different ionic species from a given analyte compound, summation of these intensities was employed to get an estimate on relative analyte concentration in the samples, assuming that the response factors are identical for all different ionic species associated with a single molecular species/analyte compound.

Since identical samples were analyzed on multiple platforms in course of training, we could assume that the concentration of a given analyte was also identical within the same anatomical segment. The cross-platform normalization factors (which would theoretically be the ratio of cumulative response factors) were thus estimated as a ratio of average intensity values of analytes measured on one platform, relative to those measured on another, since the concentration was identical.

The cross-platform normalization factors compensate for instrument-specific variation without losing tissue type specificity. More sophisticated strategies that can be used to estimate cross-platform normalization factors are described elsewhere [14-21], but the simple strategy described above was sufficient to account for platformspecific variation in our study. Figure 3 shows the symmetrical distribution of analyte-specific normalization factors between pairs of platforms in logarithmic scale, with the majority of analytes having consistent intensities between platforms. Roughly equal numbers of analytes were up- or down-scaled, the majority of which are within the range of 32 times. As scaling factors increase, their likelihood of occurrence decreases. These results indicate that a single scaling factor is not sufficient to bring all analyte intensities to a common scale between platforms; hence, analyte-specific scaling factors were used.

Application of XMS to other platforms therefore is primarily feasible if the ionization methods create a different set of ions, but ionize the same molecules. In this case, XMS together with a suitable high mass accuracy compound database is able to find a common set of compounds that can be used as variables for statistical analysis. Also, mass spectrometers should provide a sufficiently good mass accuracy and resolution to obtain a low number of false positive peak annotations. If the ionization methods are the same but different mass spectrometers are used, peak annotation step and application of scaling factors might not be necessary [39].

Changes in experimental parameters can have an impact on mass spectral patterns within one platform. In this case, XMS is not feasible as the scaling factors do not reflect the actual intensity difference anymore. However, each individual platform might be more or less stable to parameter changes in regard of mass spectral patterns. DESI, for instance, shows stable mass spectral patterns for a wide range of experimental parameter changes, such as in solvent composition, electrospray voltage, or electro-sprayer to MS-inlet capillary distance (Supplemental Information Figure S-17). Still, it is important that the user of XMS is aware of the stability of the used platform. If the mass spectral patterns do indeed change significantly, intra-platform normalization has to be carried out first (e.g., by applying XMS without peak annotation step). In this approach, XMS finds the scaling factors for two different experimental parameter setups and can then normalize new mass spectra of one platform to match the other.

\section{Cross-Platform Normalization Method Validation}

Mean-Mean Plots and Inter-Platform Concordance A successful cross-platform normalization strategy ought to preserve biologically relevant tissue information, while eliminating noise and instrumentation-related intensity differences of the ions detected by both platforms. Therefore, meanmean analyte intensity plots between two instrumental setups and one tissue type should show a population of data points along an ideal $45^{\circ}$ line (Figure 4). REIMS Orbitrap and DESI Orbitrap mean analyte intensities of porcine tissues show a high similarity with a concordance correlation coefficient of 0.91 for kidney cortex tissue. Some analytes were over- or under-scaled, which means obtained scaling factors from the training set do not properly normalize the intensities of the test set for this subset of analytes. This non-linear relationship can be caused by e.g., divergence of ion beam at higher intensities resulting in poor transmission efficiency or by ion/matrix suppression effects. However, the correlation coefficients obtained demonstrate that over- or under-scaling can be neglected. This also implies that possible sampling technique-specific ion suppression effects are mostly compensated by XMS normalization. Still, these observations may be subject of future research. REIMS Orbitrap and LDI Orbitrap data plot show a higher concordance correlation coefficient, since the intensity distribution of the analytes already show 

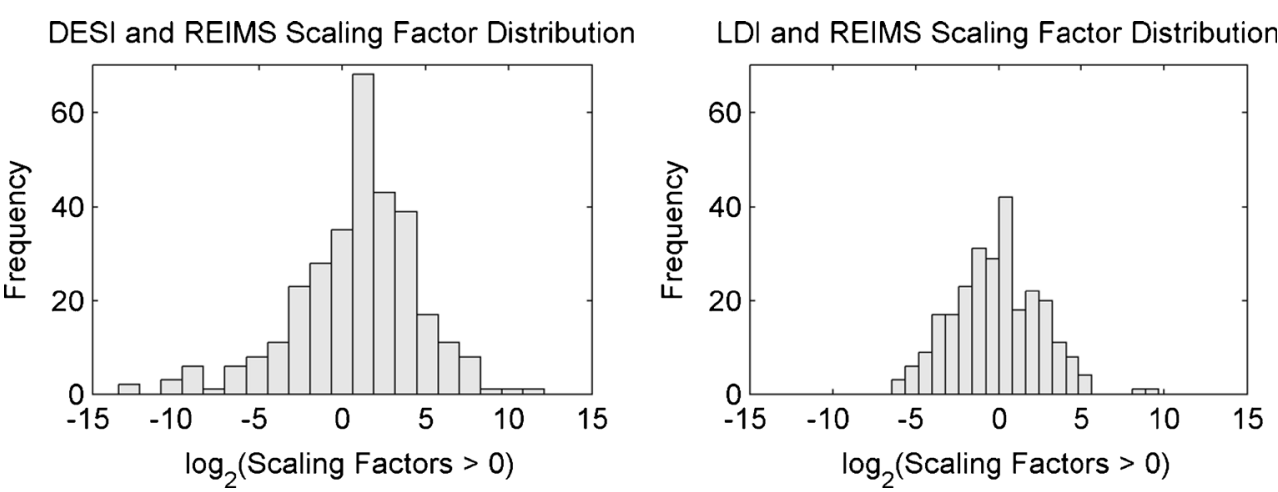

Figure 3. Distribution of analyte-specific normalization factors between platforms DESI FT-ICR versus REIMS Orbitrap $(n=312)$ and LDI Orbitrap versus REIMS Orbitrap $(n=262)$

high similarity at the raw mass spectrum level. Additional mean-mean intensity plots and concordance correlation coefficients for other tissue types demonstrate high similarities of cross-platform/instrument normalized analyte profiles within a tissue type (see Supplemental Information Figure S-6 to Figure S-8 and Table S-2). For a comparison of porcine mean-mean intensity distributions of non-normalized mass spectra and XMSnormalized mass spectra without peak annotation step, see Supplemental Information Figure S-2 to Figure S-4, and Figure S-12 to Figure S-14. It is shown that data points of non-normalized mass spectra are not populated across the ideal $45^{\circ}$ line, but indicate an intensity overbalance to one of the platforms. Mass spectra subjected to XMS without peak annotation step show similar data point populations as analyte profiles, with a large majority of data points in the low intensity range of the plots, whereas analyte profile intensities are more evenly distributed across the whole intensity range.
Unsupervised Clustering via Principal Component Analysis Although similarity between analyte profiles of individual tissue types was shown by mean-mean intensity plots, a successful cross-platform normalization method ought to also preserve the biological differences between tissue types. We used unsupervised PCA to study this tissue type-related biological variance. Without XMS normalization, the major variation seen between TIC normalized tissue mass spectral data points on the PC map was due to the different ionization methods, rather than tissue type, with the exception of brain grey matter (Figure 5a). This indicates that instrumental variation overwhelms biological variation between tissue types. In contrast, the distribution pattern of XMS normalized data corresponds to tissue types, irrespective of the ionization method and instrument used (Figure $5 \mathrm{~b}$ and d). Supplemental Information Figure S-15 shows PCA plots of mass spectra subjected to XMS without peak annotation step, indicating larger intra-platform
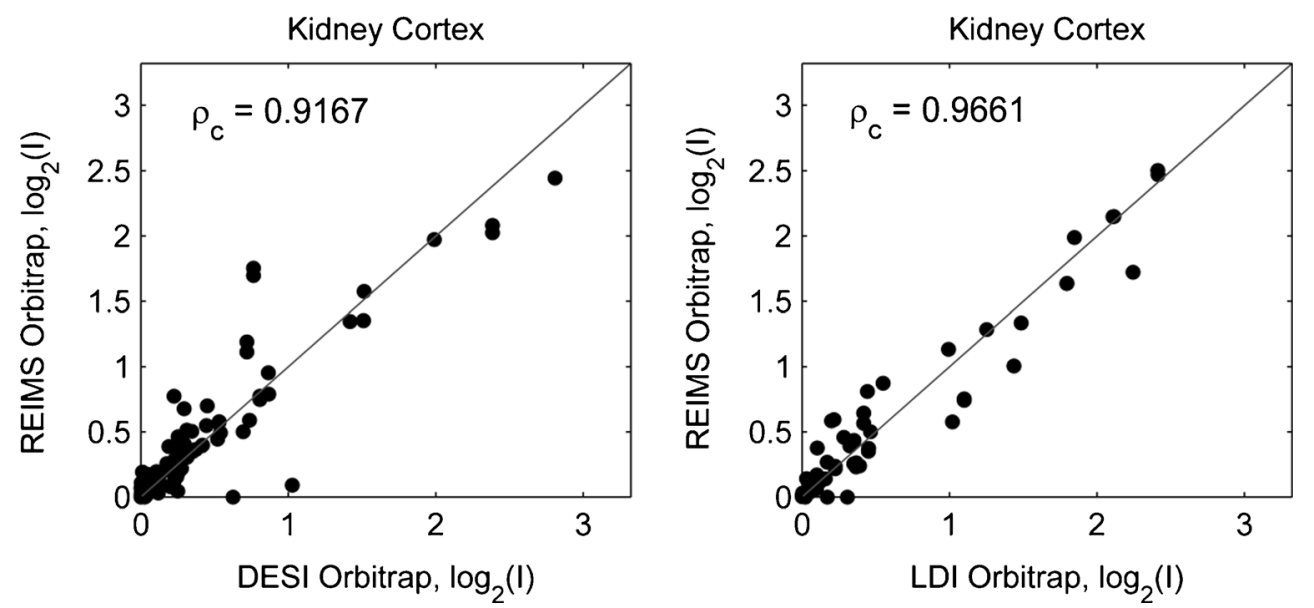

Figure 4. Mean-mean cross-platform normalized analyte profile intensity plots between different instrumental setups. $\rho_{c}=$ concordance correlation coefficient. Only analytes present in at least $10 \%$ of the mass spectra of each tissue type were included 
(a)

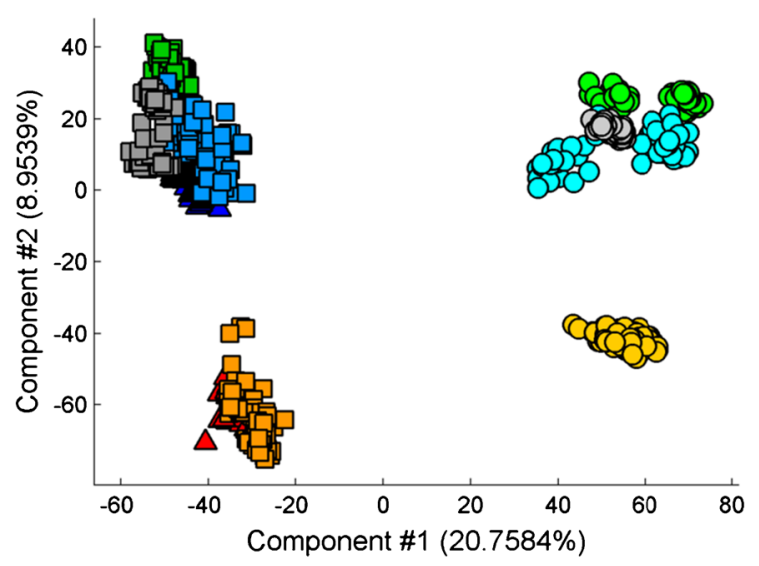

(b)

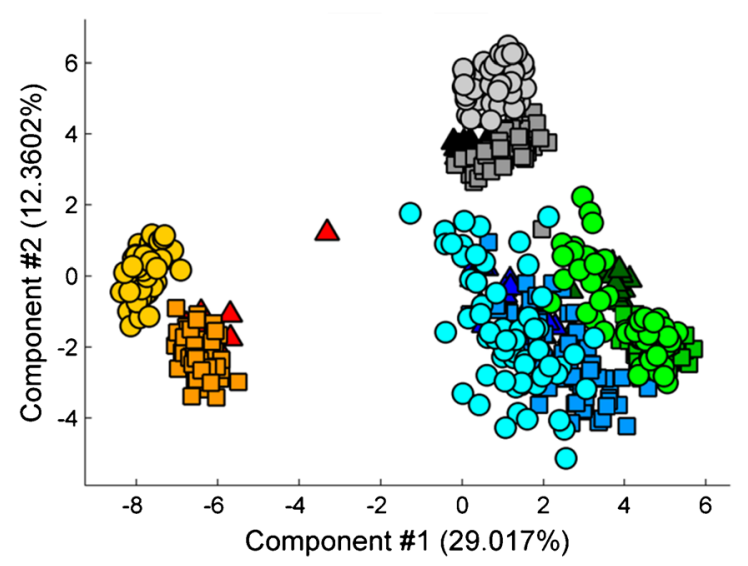
$\triangle \quad$ DESI FT-ICR Grey Matter
$\triangle$ DESI FT-ICR Kidney Cortex
$\Delta$ DESI FT-ICR Kidney Medulla
$\Delta$ DESI FT-ICR Liver

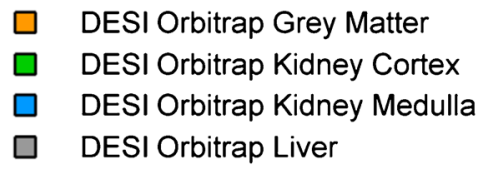
口 DESI Orbitrap Liver

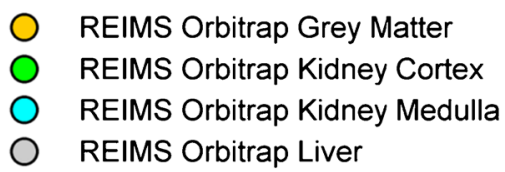

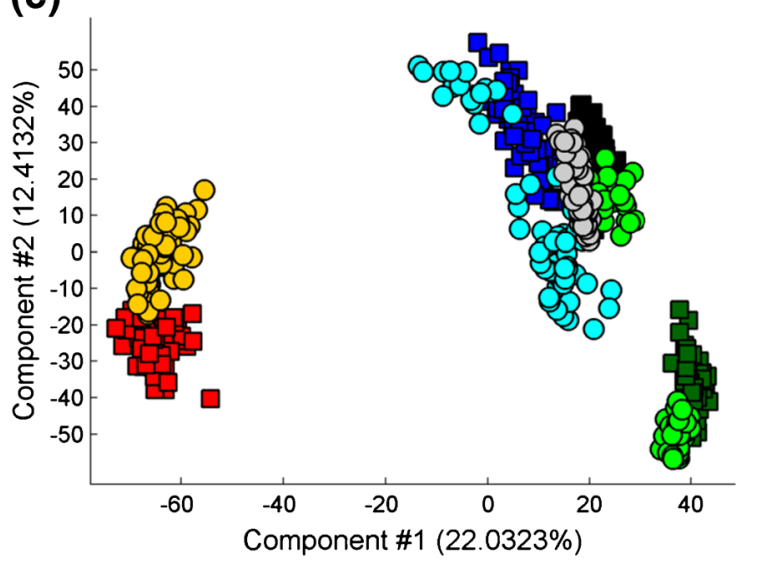

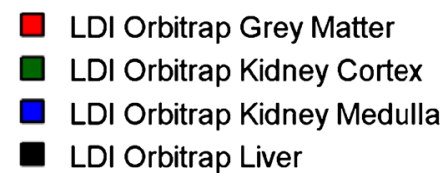

(d)

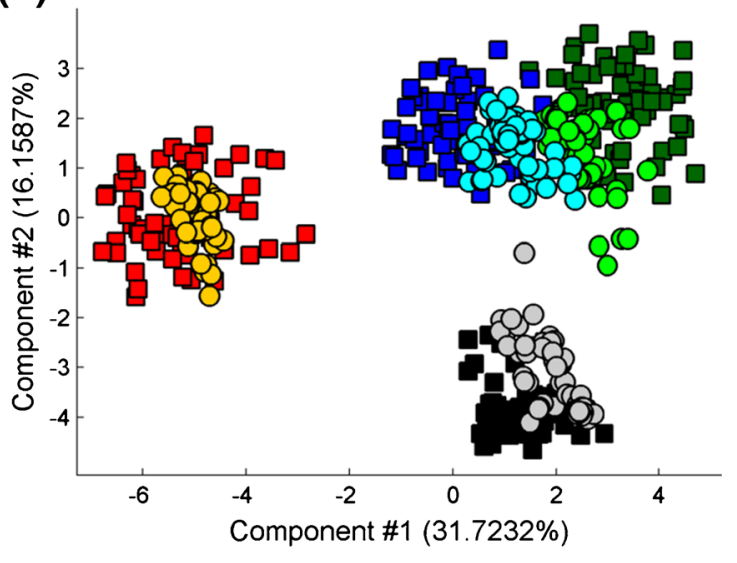

REIMS Orbitrap Grey Matter

O REIMS Orbitrap Kidney Cortex

REIMS Orbitrap Kidney Medulla

O REIMS Orbitrap Liver

Figure 5. Principal component analysis of tissue types analyzed by different instrumental setups. Percentage values are explained variance. Mass spectra in (a) and (c) were normalized to their total ion count. DESI FT-ICR was used as a reference platform in the training phase

intensity variation and more overlap of distinct tissue types.

Distance-Based k-Nearest Neighbor Classification We then applied k-nearest neighbor distance-based classification to determine the capacity of the algorithm to correctly predict porcine tissue type for data from one platform based on data obtained on a different platform. The 3-nn classification procedure was applied to REIMS Orbitrap test dataset using cross-platform normalized DESI FT-ICR and LDI Orbitrap reference datasets (Figure 6).
Without XMS normalization, the classification accuracy of REIMS data using DESI FT-ICR data was very poor $(25 \%)$, whereas the cross-platform normalized REIMS profiles were correctly classified into different tissue types with an accuracy of $98 \%$. Due to high spectral similarities between REIMS and LDI platforms, the overall classification accuracy of REIMS data using LDI data was already $98 \%$ without cross-platform normalization, and it improved to $99 \%$ after application of XMS normalization. The ability of XMS to improve kidney medulla classification accuracy by another 5\% seems marginal, but being able to optimize a very well working system even more is 


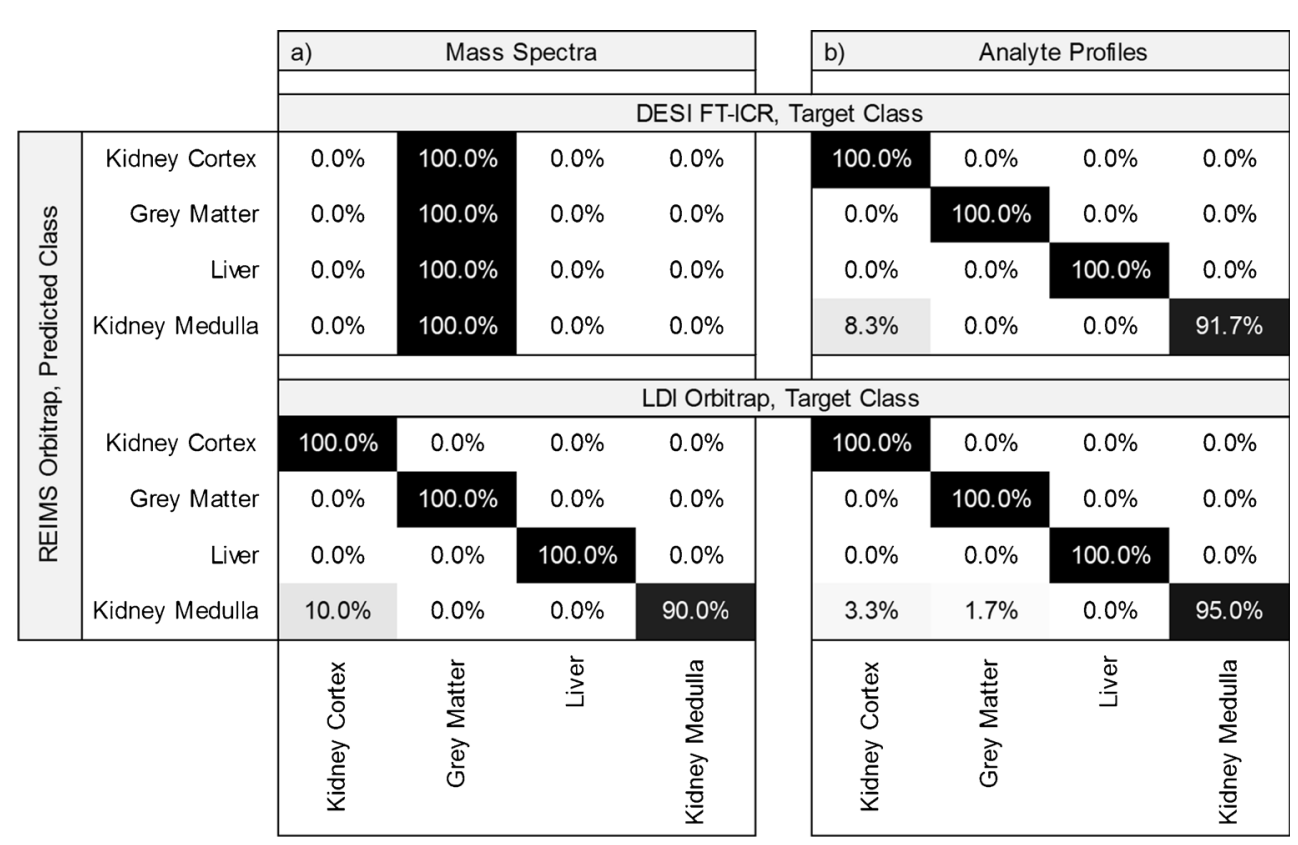

Figure 6. Performance of unknown tissue type classification based on $\mathrm{k}(3)$-nearest neighbor algorithm. Mass spectra in (a) were normalized to their total ion count. Spectra per tissue type: $n=60$

noteworthy. The lower classification accuracy for kidney medulla was mainly due to the reduced information content available as observed by a lower total ion count (see Supplemental Information Table S-1). However, we have found that the difference in total ion count between platforms has little impact on overall classification performance. This is exemplified by the fact that although DESI TIC intensity is one order of magnitude higher than that of LDI, the classification rates are comparable. Additionally, the XMS algorithm compensates for possible spectral variation caused by the several-month time difference between the acquisition of the reference and test data. Supplemental Information Figure S-16 shows classification performance for XMS applied to mass spectra without peak annotation step. For both DESI FT-ICR and LDI Orbitrap target classes, lower overall classification accuracies of $85.4 \%$ were achieved.

Analysis of human colorectal analyte profiles with SVM classifier and leave-one-patient-out cross validation achieved an overall classification accuracy of $94 \%$, whereas cross validation on mass spectrum level resulted in $43 \%$ correct classifications (Supplemental Information Figure S-18). With this colorectal dataset, two platforms with distinctively different ionization techniques (DESI - REIMS) and mass analyzers (Orbitrap - Time of Flight) were normalized to a common set of analytes that allow the correct classification of clinically relevant samples from one platform into the other.

\section{Conclusions}

MS-based tissue profiling and imaging techniques are beginning to translate into the level of clinical diagnostics that raises strict requirements regarding reproducibility of corresponding data in order to implement accurate tissue classification using MS spectral patterns, and large histologically assigned databases are required, which are likely to be acquired over many years on multiple instrumental platforms. This data can then be used for training to make newly acquired datasets comparable. The proposed XMS method offers an approach for converting raw mass spectral profiles of biological samples measured by distinct desorption ionization methods and/or instrumental setups to cross-platform normalized chemical profiles. We have demonstrated the capacity of the XMS method to sufficiently reduce analytical platform-specific variation of MS data resulting in high inter-platform concordance coefficients of analyte intensities, accurate classification of different tissue types, measured on multiple platforms, irrespective of the ionization technique or mass spectrometer used. The proposed validation strategy can also be used for further refinement of cross-platform normalization methods. The accurate tissue identification obtained on XMS normalized data could in the future allow imaging based desorption ionization methods to be used for the population of large-scale MS-based tissue databases, which are suitable for near real time intra-operative tissue identification using REIMS technology.

\section{Acknowledgments}

This work was supported by the European Research Council under Starting Grant Scheme (Grant Agreement no: 210356; DESI_JeDI_Imaging), Hessisches Ministerium für Wissenschaft und Kunst (LOEWE Schwerpunkt "AmbiProbe"), by a junior research fellowship (to K.V.) and by the European 
Union Seventh Framework Programme FP7 under grant agreements 305259 (3D Massomics) and 305940 (ISD).

\section{References}

1. Van Berkel, G.J., Pasilis, S.P., Ovchinnikova, O.: Established and emerging atmospheric pressure surface sampling/ionization techniques for mass spectrometry. J. Mass Spectrom. 43, 1161-1180 (2008)

2. Liebl, H.: Ion microprobe mass analyzer. J. Appl. Phys. 38, 5277-5283 (1967)

3. Takats, Z., Wiseman, J.M., Gologan, B., Cooks, R.G.: Mass spectrometry sampling under ambient conditions with desorption electrospray ionization. Science 306, 471-473 (2004)

4. Karas, M., Bachmann, D., Bahr, U., Hillenkamp, F.: Matrix-assisted ultraviolet-laser desorption of nonvolatile compounds. Int. J. Mass Spectrom. Ion Process 78, 53-68 (1987)

5. Caprioli, R.M., Farmer, T.B., Gile, J.: Molecular imaging of biological samples: Localization of peptides and proteins using MALDI-TOF MS. Anal. Chem. 69, 4751-4760 (1997)

6. Rompp, A., Guenther, S., Schober, Y., Schulz, O., Takats, Z., Kummer, W., Spengler, B.: Histology by mass spectrometry: Label-free tissue characterization obtained from high-accuracy bioanalytical imaging. Angew. Chem. Int. Ed. 49, 3834-3838 (2010)

7. Cornett, D.S., Reyzer, M.L., Chaurand, P., Caprioli, R.M.: MALDI imaging mass spectrometry: Molecular snapshots of biochemical systems. Nat. Methods 4, 828-833 (2007)

8. Walch, A., Rauser, S., Deininger, S.O., Höfler, H.: MALDI imaging mass spectrometry for direct tissue analysis: A new frontier for molecular histology. Histochem. Cell Biol. 130, 421-434 (2008)

9. Schwamborn, K., Caprioli, R.M.: Molecular imaging by mass spectrometry-looking beyond classical histology. Nat. Rev. Cancer. 10, 639-646 (2010)

10. Veselkov, K.A., Mirnezami, R., Strittmatter, N., Goldin, R.D., Kinross, J., Speller, A.V.M., Abramov, T., Jones, E.A., Darzi, A., Holmes, E., Nicholson, J.K., Takats, Z.: Chemo-informatic strategy for imaging mass spectrometry-based hyperspectral profiling of lipid signatures in colorectal cancer. Proc. Natl. Acad. Sci. U.S.A. 111, 1216-1221 (2014)

11. Balog, J., Szaniszlo, T., Schaefer, K.C., Denes, J., Lopata, A., Godorhazy, L., Szalay, D., Balogh, L., Sasi-Szabo, L., Toth, M., Takats, Z.: Identification of biological tissues by rapid evaporative ionization mass spectrometry. Anal. Chem. 82, 7343-7350 (2010)

12. Rompp, A., Guenther, S., Takats, Z., Spengler, B.: Mass spectrometry imaging with high resolution in mass and space (HR(2) MSI) for reliable investigation of drug compound distributions on the cellular level. Anal. Bioanal. Chem. 401, 65-73 (2011)

13. Angel, P.M., Spraggins, J.M., Baldwin, H.S., Caprioli, R.: Enhanced sensitivity for high spatial resolution lipid analysis by negative ion mode matrix assisted laser desorption ionization imaging mass spectrometry. Anal. Chem. 84, 1557-64 (2012)

14. Rudy, J., Valafar, F.: Empirical comparison of cross-platform normalization methods for gene expression data. BMC Bioinforma. 12, 467 (2011)

15. Shabalin, A.A., Tjelmeland, H., Fan, C., Perou, C.M., Nobel, A.B...: Merging two gene-expression studies via cross-platform normalization. Bioinformatics 24, 1154-1160 (2008)

16. Benito, M., Parker, J., Du, Q., Wu, J., Xiang, D., Perou, C.M., Marron, J.S.: Adjustment of systematic microarray data biases. Bioinformatics 20, 105114 (2004)

17. Walker, W., Liao, I., Gilbert, D., Wong, B., Pollard, K., Mcculloch, C., Lit, L., Sharp, F.: Empirical Bayes accommodation of batch-effects in microarray data using identical replicate reference samples: Application to RNA expression profiling of blood from Duchenne muscular dystrophy patients. BMC Genomics 9, 494 (2008)

18. Martinez, R., Pasquier, C., Pasquier, N.: GenMiner: Mining informative association rules from genomic data. Proc (IEEE Int Conf Bioinformatics Biomed.) 1, 15-22 (2007)

19. Xia, X., Mcclelland, M., Porwollik, S., Song, W., Cong, X., Wang, Y.: WebArrayDB: Cross-platform microarray data analysis and public data repository. Bioinformatics 25, 2425-2429 (2009)
20. Bolstad, B., Irizarry, R., Astrand, M., Speed, T.: A comparison of normalization methods for high density oligonucleotide array data based on variance and bias. Bioinformatics 19, 185 (2003)

21. Lacson, R., Pitzer, E., Kim, J., Galante, P., Hinske, C., OhnoMachado, L.: DSGeo: Software tools for cross-platform analysis of gene expression data in GEO. J. Biomed. Inform. 43, 709-715 (2010)

22. Wang, W., Zhou, H., Lin, H., Roy, S., Shaler, T.A., Hill, L.R., Norton, S., Kumar, P., Anderle, M., Becker, C.H.: Quantification of proteins and metabolites by mass spectrometry without isotopic labeling or spiked standards. Anal. Chem. 75, 4818-4826 (2003)

23. Hamm, G., Bonnel, D., Legouffe, R., Pamelard, F., Delbos, J.-M., Bouzom, F., Stauber, J.: Quantitative mass spectrometry imaging of propranolol and olanzapine using tissue extinction calculation as normalization factor. J. Proteome 75, 4952-4961 (2012)

24. Liu, X., Dekker, L.J.M., Wu, S., Vanduijn, M.M., Luider, T.M., Tolić, N., Kou, Q., Dvorkin, M., Alexandrova, S., Vyatkina, K., Paša-Tolić, L., Pevzner, P.A.: De novo protein sequencing by combining top-down and bottom-up tandem mass spectra. J. Proteome Res. 13, 3241-3248 (2014)

25. Herzog, R., Schwudke, D., Schuhmann, K., Sampaio, J.L., Bornstein, S.R., Schroeder, M., Shevchenko, A.: A novel informatics concept for highthroughput shotgun lipidomics based on the molecular fragmentation query language. Genome Biol. 12, R8 (2011)

26. Hartler, J., Trötzmüller, M., Chitraju, C., Spener, F., Köfeler, H.C., Thallinger, G.G.: Lipid data analyzer: Unattended identification and quantitation of lipids in LC-MS data. Bioinformatics 27, 572-577 (2011)

27. Press, W.H.: Numerical Recipes: The Art of Scientific Computing, 3rd edn. Cambridge University Press, Cambridge (2007)

28. Lin, L.: A concordance correlation coefficient to evaluate reproducibility. Biometrics 45, 255-268 (1989)

29. Owen, A.B..., Perry, P.O.: Bi-Cross-Validation of the SVD and the nonnegative matrix factorization. Ann. Appl. Stat. 3, 564-594 (2009)

30. Chang, C.-C., Lin, C.-J.: LIBSVM: A library for support vector machines. ACM Trans. Intell. Syst. Technol. 2, 1-27 (2011)

31. Kessner, D., Chambers, M., Burke, R., Agusand, D., Mallick, P.: ProteoWizard: Open source software for rapid proteomics tools development. Bioinformatics 24, 2534-2536 (2008)

32. Takats, Z., Wiseman, J.M., Cooks, R.G.: Ambient mass spectrometry using desorption electrospray ionization (DESI): Instrumentation, mechanisms, and applications in forensics, chemistry, and biology. J. Mass Spectrom. 40, 1261-1275 (2005)

33. Venter, A., Sojka, P.E., Cooks, R.G.: Droplet dynamics and ionization mechanisms in desorption electrospray ionization mass spectrometry. Anal. Chem. 78, 8549-8555 (2006)

34. Schafer, K.C., Denes, J., Albrecht, K., Szaniszlo, T., Balog, J., Skoumal, R., Katona, M., Toth, M., Balogh, L., Takats, Z.: In vivo, in situ tissue analysis using rapid evaporative ionization mass spectrometry. Angew. Chem. Int. Ed. 48, 8240-8242 (2009)

35. Balog, J., Sasi-Szabo, L., Kinross, J., Lewis, M.R., Muirhead, L.J., Veselkov, K., Mirnezami, R., Dezso, B., Damjanovich, L., Darzi, A., Nicholson, J.K., Takats, Z.: Intraoperative tissue identification using rapid evaporative ionization mass spectrometry. Sci. Transl. Med. 5, 194ra93 (2013)

36. Gerbig, S., Golf, O., Balog, J., Denes, J., Baranyai, Z., Zarand, A., Raso, E., Timar, J., Takats, Z.: Analysis of colorectal adenocarcinoma tissue by desorption electrospray ionization mass spectrometric imaging. Anal. Bioanal. Chem. 403, 2315-2325 (2012)

37. Kind, T., Fiehn, O.: Seven Golden Rules for heuristic filtering of molecular formulas obtained by accurate mass spectrometry. BMC Bioinformatics $\mathbf{8}$, 105 (2007)

38. Kind, T., Fiehn, O.: Metabolomic database annotations via query of elemental compositions: Mass accuracy is insufficient even at less than $1 \mathrm{ppm}$. BMC Bioinformatics 7, 234 (2006)

39. Strittmatter, N., Rebec, M., Jones, E.A., Golf, O., Abdolrasouli, A., Balog, J., Behrends, V., Veselkov, K.A., Takats, Z.: Characterization and identification of clinically relevant microorganisms using rapid evaporative ionization mass spectrometry. Anal. Chem. 86, 6555-6562 (2014) 